УДК 81

DOI: 10.33184/NYVB-2021-04-06.7

Wotjak, Gerd (Prof. Dr. Leipzig, Deutschland)

\title{
VERB IM HEUTIGEN DEUTSCH
}

1. Im Verb liegt die Verbindungslinie zwischen der Syntax und Morphologie, zwischen der Syntax und Semantik. Diese syntaktische wie semantische Aspekte aufeinander beziehenden Angaben weisen mit den bereits viel früher ausgearbeiteten Satzbauplänen, deutliche Berührungspunkte auf, die insofern herausgestellt zu werden verdienen, weil sie unabhängig voneinander postuliert wurden und dabei doch sehr weitgehende Übereinstimmungen (wohl doch gegenstandsbedingt, dabei nicht zufällig verbzentriert) aufweisen. Auch hier zeigen sich Paradigmen überschreitende Konvergenzen, die uns optimistisch stimmen sollten dahingehend, dass auch eine weiterführende Annäherung unterschiedlicher Herangehensweisen möglich sein sollte. Aus romanistischer Sicht sei hier bspw. nur auf das berühmt gewordene petit drame von Tesnière (1959) verwiesen, aber auch auf die syntaktisch-semantischen Satzschemata, wie sie von V. Báez San José zunächst allein, dann in elaborierterer Form und auf der Grundlage computergestützter Analysen von ca. 7000 spanischen Verben zusammen mit I. Penadés Martínez (1990) vorgestellt wurden. Dabei dürften nach einer auf der internationalen Tagung zu Grammatik und Lexikon in Lugo im September 2000 von Báez San José mündlich geäußerten Überzeugung maximal 700 solcher Satzschemata (nach ihm seien es 672 unter Einbezug aktiver wie passiver Verwendung) ausreichend dafür sein, um die syntagmatisch-syntaktische wie semantische (sememotaktische) Kombinatorik des gesamten spanischen Verbinventars in aktivischer wie passivischer Verwendung abzudecken.

2. Durch das Bestreben, die lexikalisch-semantische Beschreibung passfähig zu machen für den Prädikatsrahmen Diks, wird in der Functional Lexicology im Übrigen - auch dies keineswegs so neu wie man annehmen könnte - auch die von der Lexematik, aber auch anderen Beschreibungen der lexikalischen Bedeutung, vernachlässigte Schnittstelle von Semantik und Syntax thematisiert. Dabei lassen sich nicht zufällig bei der Bedeutungsbeschreibung von Verben - wie etwa 
ein von E. Coseriu in einer Diskussion auf dem Münsteraner Lexikologiekolloquium 2000 erwähntes und von uns aufgegriffenes Beispiel unter (1) zeigt - solche Bedeutungsbeschreibungen durch geringfügige Ergänzungen bzw. Umformulierungen mehr oder minder deutlich passgerecht machen für die Angabe von Argumentstrukturen/ Argumentpotenzialen (Wotjak, 1984, 1986, 1997, 2006).

3. Es wäre schade, wenn diese beachtliche Deckungsgleichheit nicht bewusst ausgenutzt würde, um die vorhandenen Überschneidungen zwischen lexematischer Herangehensweise und weiteren modernen semantischen Ansätzen $\mathrm{zu}$ verdeutlichen, bestehende Abschottungen $\mathrm{zu}$ überwinden und bspw. auch die Semantik-Syntax-Schnittstelle an die Beschreibung des syntagmatischkombinatorischen Potenzials der Verbbeschreibungen anzukoppeln. Dabei wären in einem hier nicht nachvollzogenen Vorgehen im Zusammenhang mit einer Valenzbeschreibung bspw. ganz im Sinne des Jubilars 1995 auch die Beziehungen zur funktionalen Satzperspektive herauszuarbeiten und den Spezifika der Pro-Drop-Sprachen Portugiesisch, Spanisch und auch Italienisch mit dem in ihnen üblichen Verzicht auf die lexikalisch-pronominale Besetzung der Subjektposition ebenfalls ausdrücklich Rechnung zu tragen (dazu bereits Koller 1982, d. h. sehr früh).

4. Aus germanistischer Sicht wäre vor allem auf die Satzschemata (syntaktische wie semantische) in den Grammatiken von Helbig/Buscha (ab 1984), auf Ulrich Engels Grammatikbeschreibungen (1988), aber auch auf die dreibändige IdS-Grammatik verwiesen und als leider stark in Vergessenheit geratenen Analysten deutscher Satzmodelle auf Bernhard Engelen $(1968,1975)$ sowie nicht zuletzt auch auf Wilhelm Bondzios Funktorenstrukturen (1994), von denen - dem Nestor der semantisch-logischen Valenzbeschreibung zufolge - gut 150 ausreichen würden, um als inhaltliche Bezugspunkte für alle denkbaren deutschen Verb-Bedeutungen/Verb-LE zu fungieren.

In dem Kontext müssen aber auch die Analysen der um Maurice Gross, inzwischen vor allem Gaston Gross und das L.A.D.L. zentrierten, gleichermaßen - wohl nicht zufällig - eng mit der Computernutzung verbundenen Untersuchungen der sogenannten lexique-grammaire erwähnt werden. Deren hauptsächlich aufs Französische konzentrierten Analysen von ca. 15.000 Verben nehmen allerdings nicht auf die durch 
die lexikalische Bedeutung der Verben bedingten lexikalischen Varianten Rücksicht. Sie liefern vielmehr eine globale, undifferenzierte Distributionsanalyse der jeweiligen polysemen Verbvorkommen, in die neben tatsächlichen Textwortokkurrenzen/ Konkordanzen auch zusätzliche Angaben, bspw. zur Imperativfähigkeit (also Transformationen im Sinne von Harris), mit aufgenommen wurden. Im Ergebnis sind beeindruckende synoptische Darstellungen zum Verwendungspotenzial der einzelnen Verben entstanden, wobei als Quintessenz die eher demoralisierende Feststellung steht, dass faktisch jede Verbform eine sich zumindest minimal unterscheidende idiosynkratische Distribution/syntagmatische Kombinatorik und Verwendungsrestriktion aufweist.

5. Bei der Beschreibung des semantischen Kombinationspotenzials der Verben als intrinsische Sememotaktik bzw. Kollokabilitätsvorgabe sind neben Argumentvariablen, den Leerstellenvorgaben $=$ Slots, auch Argumentkonstanten $\mathrm{zu}$ beachten. Dabei sind für die Argumentleerstellen/Slots semantisch-funktionale (= semantische Kasus oder thematische bzw. Thetarollen) wie semantisch-kategoriale (klassematische) Charakterisierungen vorzunehmen, denen die konkreten LE als Leerstellenbesetzungen/Filler und Aktanten der morphosyntaktischen Kombinatorik des Verbs gerecht werden müssen. Für die Argumentkonstanten dagegen würden semantisch-funktionale Bestimmungen ausreichen, weil die semantisch-denotativen (kategorialen) Angaben ja mit der konkreten lexikalischen Einheit/LE gegeben sind, die als intralexematische (inkorporierte) oder intrasememische Argumentkonstante fungiert (dazu Wotjak, 1991b, 2006). Wir sprechen auf der semantischen Ebene von einem Argumentenpotenzial bzw. einer Argumentstruktur, der auf der morphosyntaktischen Ebene die Wertigkeit als Aktantenpotenzial entspricht, wobei auch von einer Aktantifizierung/= Besetzung der Argumentenslots durch Filler gesprochen werden kann, syntaktische Charakteristika der Distribution/Kollokabilität also als aufs Engste mit der Semantik verknüpfte und letztlich durch diese prädeterminierten Erscheinungen verstanden werden.

6. Welche der Argumentvorgaben (= Variablen, Slots) tatsächlich aktantifiziert werden, hängt zum einen von der idiosynkratischen Verbaktanz, d. h. dem unverzichtbaren Minimum der Kopräsenz von Aktanten bei der Verbverwendung im konkreten Äußerungskontext, 
zum anderen von Erfordernissen der kommunikativen Relevanzgebung (vgl. auch Koller 1995) ab. Dabei macht es Sinn, präferierte bzw. blockierte, d. h. nicht ausgenutzte bzw. nicht nutzbare Aktantenkombinationen im Sinne von Aktantensequenzen herauszustellen.

(4) Aktantensequenzen für bezahlen:

Subj + Verb $\rightarrow$ Vater hat (schon) bezahlt .

Subj + Verb + Od/THEMA $\rightarrow$ Vater hat schon alle Arbeitsstunden bezahlt.

Subj + Verb + Od/ADRESSAT $\rightarrow$ Vater hat den Handwerker schon bezahlt.

Subj + Verb + Od/ADRESSAT + p1S/THEMA $\rightarrow$ Vater hat den Handwerker für seine Arbeit bezahlt.

Subj + Verb + Oi/ADRESSAT + Od/INSTRUMENT + p1S/THEMA $\rightarrow$ Vater hat dem Handwerker für seine Arbeit 40 DM bezahlt.

$\mathrm{Subj}+\mathrm{Verb}+\mathrm{p} 1 \mathrm{~S} / \mathrm{THEMA} \rightarrow$ Vater hat für die Arbeit doch schon einmal bezahlt.

Vater hat für seine Vertrauensseligkeit teuer bezahlen müssen.

$*$ Subj + Verb + Oi $\rightarrow$ für das Deutsche blockierte Kombinationen

$$
* \mathrm{Subj}+\mathrm{Verb}+\mathrm{Oi}+\mathrm{p} 1 \mathrm{~S}
$$

Dabei werden die Aktanten für die romanischen Sprachen syntaktischfunktional als Subjekt, direktes und/oder indirektes Objekt, präpositives Objekt bestimmt, wobei zumindest in einigen romanischen Sprachen die Subjektposition unbesetzt bleiben kann (dazu u. a. Koller, 1982). Für das Deutsche als morphologisch flektierende Sprache könnte sich daneben auch eine Angabe bspw. der Kasusformen als sinnvoll erweisen; so in den Valenzwörterbüchern bei Helbig/Schenkel (1969 ff.) als $S n, S d, S a, S g, p S$, Inf, NSdass etc. (vgl. 4).

Es sei in diesem Kontext auch darauf aufmerksam gemacht, dass sich die Charakteristik der Verbmitspieler durchaus zwischen den Anhängern der Leipziger und der Mannheimer Schule unterscheidet, wobei neben Aktanten auch Zirkumstanten in einem hier nicht detaillierter zu bestimmenden Sinne als zulässige bzw. notwendige Ergänzungen aufgeführt werden und durchaus nicht alle Beschreibungen sensibilisiert erscheinen gegenüber einer Unterscheidung in Ergänzungen und (Freie) Angaben, Komplemente und Adjunkte bzw. dafür verwertbare Unterscheidungskriterien 
angeführt haben. Zweifellos wäre die Erörterung dieser für die Valenzbeschreibung außerordentlich wichtigen Problematik Gegenstand eines eigenen Beitrags.

7. Immerhin sei an dieser Stelle darauf hingewiesen, dass die Functional Lexicology zwar praktische Belege für solche Adjunkte liefert, die Unterscheidung in Argumente und Modifikatoren (satellites) jedoch kaum theoretisch problematisiert. Dass diese Unterscheidung bei einer lexematischen Analyse nach dem traditionellen Verständnis außen vor bleibt kann dagegen nicht wundern, da in der Lexematik ja die für die Designation relevanten Argumentvorgaben des feldkonstituierenden gemeinsamen Archisemems (vgl. dazu die Ausführungen zu den Generischen und Spezifischen Archisemformeln in B.Wotjak/G.Wotjak 1995) unbeachtet bleiben. Herausgestellt $\mathrm{zu}$ werden verdient aber in diesem Kontext, dass gerade der Rekurs auf lexematische Grundprinzipien und Methoden, bspw. die distinktiv-oppositive Merkmalsermittlung im Rahmen von Feldern, in Gestalt der von der modernen Semantik häufig genug unberücksichtigten Differentiaseme letztlich durchaus Modifikatorseme herauszuarbeiten erlaubt.

8. Hinsichtlich der uns im Hinblick auf die Functional Lexicology/FL und die Zweiebenensemantik besonders interessierenden Semantikbeschreibung sollte im Vorfeld noch auf zwei z. T. nur wenig beachtete, aber sehr interessante Ansätze verwiesen werden; zum einen auf die logisch-semantische Valenzbeschreibung (Bondzio, 1994) und (Welke, 1988) sowie auf die modernere Ansätze zu einer kombinierten Beschreibung vereinigenden Untersuchungen (Kunze, 1991, 1993), bei der eine deduktive Ableitung von Sememrepräsentationen aus den Feldoberbegriffen, einschließlich emphatisch gewichteter Prädikate und Argumente vorgeführt wird. Dabei sind wir uns bewusst, dass die Funktorenstrukturen in den Generischen bzw. auch den Spezifischen Archisemformeln (B.Wotjak/G.Wotjak, 1995) nachweisbar, manifestiert sind, doch erscheinen letztere weniger apodiktischdeduktiv festgelegt, sondern bspw. auch aus Wörterbuchdefinitionen im Sinne der durch die Functional Lexicology bevorzugten Vorgehensweise ableitbar.

Nach unserer bisherigen aufrissartigen Darstellung konkurrierender und partiell kongruierender /konvergierender Beschreibungsansätze für die Untersuchung des Interface von Syntax und Semantik bei Verben, bei denen auch - im Einzelnen durchaus unterschiedlich intensiv und 
explizit - Fragen der Interrelation von Bedeutung und Kognition berührt werden, nun insgesamt relativ knapp und eher mit der Absicht der Herausarbeitung von Übereinstimmungen und vor allem von Unterschieden und weniger mit dem Ziel einer detaillierteren Präsentation der Vorgehensweise, nachstehend noch einige Ausführungen zur Functional Lexicology und abschließend zur Zweiebenensemantik machen.

8. Unter der Bezeichnung Functional Lexicology/FL können im Wesentlichen zwei Tendenzen/Typen von Untersuchungen zusammengefasst werden: stärker dogmatisch auf die vor allem von Leocadio Martín Mingorance (1998) formulierten Grundprinzipien eingeengte Untersuchungen durch seine Schüler mit z. T. erst nach dessen Tod vollendeten Promotionsschriften; mehr oder minder stark um lexikologische Beschreibungen bemühte Untersuchungen, die - wie auch die erwähnten Forscher - sich auf Diks vorrangig syntaxzentrierte Untersuchungen berufen. Wenn wir im Folgenden von FL sprechen, so nehmen wir dabei vor allem auf die unter a) subsumierten Analysen Bezug. Dennoch kann auch festgehalten werden, dass:

(i) alle Forscher davon ausgehen, dass die vorgelegten komplementären Untersuchungen des Lexikons vor allem den semantischen wie auch pragmatischen Aspekten dieses lexikalischen Inventars, hier zuvörderst Verben, gewidmet sein müssen, wobei deren Ergebnisse sich möglichst problemlos in die von Dik vorgegebene syntaktische Mehrebenenbeschreibung, insbesondere in die des predicate frame, integrieren bzw. mit diesem als Ergänzung/ Spezifikationen kombinieren lassen sollen;

(ii) die morphosyntaktischen und in Ansätzen auch semantischen Vorgaben dieses Prädikatsrahmens grundsätzlich als ergänzungsbedürftig angesehen werden, wobei der bei Dik nur postulierte dekompositionelle Gesichtspunkt bei Vertretern von a) durch die Einbeziehung von Ergebnissen einer lexematischen Beschreibung ganz im Sinne Coserius ausgestaltet und verwirklicht werden sollte, bei übrigen Vertretern dagegen durch Beschreibungen, die auch auf andere Paradigmen rekurrieren.

Dabei gehen die Forscher unter a) von konkreten Wörterbuchangaben aus, werden solche Verben selektiert, die ein Mindestmaß an Bestandteilen der Bedeutungsparaphrasen gemeinsam haben. Dabei fungiert in der Regel ein Archilexem mit seinem Archisemem als 
rekurrenter Teil der einzelnen Paraphrasen, weisen letztere darüber hinaus aber sich voneinander mehr oder minder stark unterscheidende weitere Merkmale/Bestimmungselemente auf. Im Unterschied zur häufig auch zu unrecht kritisierten Komponentenanalyse der europäischen strukturellen Semantik (dazu u. a. Geckeler, 1981; Kleiber, 1990; Coseriu, 1982, 1995) verzichtet die Functional Lexicology auf eine spezielle Symbolisierung dieser divergierenden Bedeutungsparaphrasenkomponenten als semantische Merkmale, als Seme (dazu detailliert Lorenz/Wotjak, 1977), ohne deshalb auf die Herausarbeitung signifikanter Unterschiede zu verzichten.

(iii) Nicht immer wird klar herausgestellt, dass die den Prädikatsrahmen bildenden Argumente wohl vor allem bis ausschließlich über diesen gemeinsamen Hauptnenner der Generischen oder Speziellen Archisemformel gewonnen werden können, wobei die Bedeutungsparaphrasen im Allgemeinen selbst eine Angabe wie die von Báez San José vorgenommene, also bspw. alguien-algo-a alguien, vermissen lassen, diese Angaben jedoch auf der syntaktischen Ebene des Prädikatsrahmens nach Dik zumindest partiell (als semantischfunktionale Kasusangaben) mitgeliefert werden.

\section{LITERATUR}

1. Buscha, Joachim / Gerhard Helbig Deutsche Grammatik: Ein Handbuch für den Ausländerunterricht, Leipzig, 1986.

2. Engel, Ulrich (1988), Deutsche Grammatik, Heidelberg

3. Eugenio Coseriu (1982), „Pour et contre l'analyse sémique“, Preprints of the Plenary Session Papers. XIIIth International Congress of Linguists, Tokyo. S. 117-129.

4. Eugenio Coseriu (1990), „Semántica estructural y semántica <<cognitiva>>", in Francisco Marsá, Jornadas de Filologíe, Universitat de Barcelona, S. 239-282.

5. Geckeler, Horst (1981), „Progrès et stagnation en sémantique structurale“, in H. Geckeler / B. Schlieben-Lange / J. Trabant y H. Weydt (eds.), Logos semantikos, vol. III, Madrid. Gredos, S. 53-69.

6. Helbig, Gerhard (1992), Probleme der Valenz- und Kasustheorie, Tübingen, Niemeyer.

7. Kleiber, G. (1990), La sémantique $d u$ prototype, Paris, Klincksieck. 
8. Koller, Erwin (1982), „Zum Subjektpronomen aus kontrastiver Sicht. Deutsch-Portugiesisch“, Sprachwissenschaft 7, S. 149-167.

9. Koller, Erwin (1995), „Valenz und funktionale Satzperspektive“, L. Eichinger / H.-W. Eroms (eds.), Dependenz und Valenz, Beiträge zur germanistischen Sprachwissenschaft 10, Hamburg, S. 105-116.

10. Lorenz, Wolfgang / Gerd Wotjak (1977), Zum Verhältnis von Abbild und Bedeutung, Berlin, Akdemie-Verlag.

11. Martín Mingorance, Leocadio (1998), El modelo lexemáticofuncional, Granada, Universidad de Granada.

12. Welke, Klaus (1988), Einführung in die Valenz- und Kasustheorie, Leipzig, Enzyklopädie.

13. Wotjak, Barbara / Gerd Wotjak (1995), „Werben für Verben?“ in Heidrun Popp (ed.), An den Quellen eines Faches. München, iudicium, S. 235-286.

14. Wotjak, Gerd (1970), „Zur semantischen Struktur deutscher Verben des Besitzens und Besitzwechsels“, Linguistische Arbeitsberichte (LAB) 3, Leipzig, S. 58-72.

15. Wotjak, Gerd (1975), „Valenzmodelle und semantische Merkmalanalyse“, Linguistische Arbeitsberichte (LAB) 11, Leipzig, S. 19-39.

16. Wotjak, Gerd (1991), „Einige Ergänzungen und Angaben zu Ergänzungen und Angaben“, in Peter Koch / Thomas Krefeldt (eds.), Connexiones Romanicae, Tübingen, Niemeyer, S. 109-128.

17. Wotjak, Gerd (1997), ,Zu den Beziehungen zwischen semantischer Mikrostruktur und morphosyntaktischer Distribution und Valenz am Beispiel deutscher Verben", in Martine Dalmas / Roger Sauter (eds.), Grenzsteine und Wegweiser, Tübingen, Stauffenburg, S. 3346.

18. Wotjak, Gerd (2006), Las lenguas, ventanas que dan al mundo, Salamanca, Universidad de Salamanca, Servicio de Publicaciones.

(C) Wotjak, Gerd, 2021 г. 